\title{
Contextos
}

\section{Arquitecturas colectivas y participación como estrategias para la construcción de la ciudad latinoamericana}

\author{
Collective architecture and participation as strategies for the construction of Latin American cities \\ Arquiteturas coletivas e participação como estratégias para construir a cidade latino-americana
}

\section{Myriam Stella Díaz-Osorio \\ Universidad Católica de Colombia, Bogotá (Colombia) \\ Facultad de Diseño - Programa de Arquitectura}

Díaz-Osorio, M. (2019). Arquitecturas colectivas y participación como estrategias para la construcción de la ciudad latinoamericana. Revista de Arquitectura (Bogotá), 21(2), 3-II. doi: http://dx.doi.org/l0.14718/ RevArq.2019.21.2.2670

\section{(†) $\$$}

http://dx.doi.org'10.14718/RevArr.2019.21.2.267
Arquitecta, Universidad Nacional de Colombia. Magíster en Historia y Teoría del Arte, la Arquitectura y la Ciudad, Universidad Nacional de Colombia.

Editora de la sección Cultura y Espacio Urbano, Revista de Arquitectura (Bogotá).

${ }_{\text {https://scholar.google.es/citations?user }=4 \mathrm{Q} 0}$ ElcAAAA|\&hl=es\&oi=sra

(D) http://orcid.org/0000-0002-0577-915 |

msdiaz@ucatolica.edu.co

\section{Resumen}

Derivado del crecimiento acelerado de las periferias latinoamericanas, en donde se hace evidente la desconexión con los procesos de planeación y la escasa participación de los arquitectos, resulta necesario identificar las tendencias relacionadas con los procesos de participación y construcción de arquitecturas colectivas. Por medio de un ejercicio comparativo entre grupos de arquitectos latinoamericanos reconocidos en seminarios y concursos en los últimos veinte años y que han realizado intervenciones en zonas periféricas de América Latina, se identifican los intereses y las operaciones comunes, entre las que se destacan la participación comunitaria, la acción interdisciplinaria, así como la gestión y la realización de proyectos de bajo costo y con materiales locales. Estas corrientes emergentes abren diversos espacios de indagación en torno a la práctica profesional. Estos aportes fortalecen el interés disciplinar por incidir en los procesos de transformación urbana que mejoran los modos de habitar y la calidad de vida de los grupos sociales. Igualmente, se identifican posibles aportes a los compromisos de la Nueva Agenda Urbana.

Palabras clave: arquitectura participativa; asentamientos humanos; bienales de arquitectura, investigación-creación; nueva agenda urbana; periferia urbana; segregación socioespacial.

\section{Abstract}

As a result of the rapid growth of Latin American peripheries, where the disconnection between planning processes and the scarce participation of architects is evident, it is necessary to identify trends related to processes of participation and construction of collective architectures. Through a comparative exercise between groups of recognized Latin American architects in seminars and competitions in the past twenty years, and who have undertaken interventions in peripheral areas of Latin America, common interests and operations have been identified, including community participation, interdisciplinary action, and the management and realization of low-cost projects with local materials. These emerging trends open diverse research spaces regarding the professional practice. These contributions strengthen the disciplinary interest in impacting processes of urban transformation that improve the ways of living and the quality of life of social groups. Additionally, the article identifies possible contributions to the commitments of the New Urban Agenda.

Keywords: Participatory architecture, human settlements, architecture biennials, researchcreation, new urban agenda, urban periphery, socio-spatial segregation.

\section{Resumo}

Com o crescimento acelerado das periferias latino-americanas, onde se torna evidente a desconexão com os processos de planejamento e a escassa participação dos arquitetos, faz-se necessário identificar as tendências relacionadas com os processos de participação e com a construção de arquiteturas coletivas. Por meio de um exercício comparativo entre grupos de arquitetos latino-americanos reconhecidos em seminários e concursos nos últimos 20 anos e que realizaram intervenções em periferias latino-americanas, são reconhecidos os interesses e as operações comuns, entre as quais são destacadas a participação comunitária, a ação interdisciplinar, a gestão e a realização de projetos de baixo custo e com materiais locais. Essas correntes emergentes abrem diversos espaços de questionamento sobre a prática profissional. Essas contribuições fortalecem o interesse disciplinar por incidir nos processos de transformação urbana que melhoram os modos de habitar e a qualidade de vida dos grupos sociais. Além disso, são identificadas possíveis contribuições para os compromissos da Nova Agenda Urbana.

Palavras-chave: arquitetura participativa; assentamentos humanos; bienais de arquitetura, pesquisa-criação; nova agenda urbana; periferia urbana; segregação socioespacial.

$$
\text { Evaluado: junio } 19 \text { / } 2019
$$

\section{Introducción}

Para la Revista de Arquitectura (Bogotá) y su compromiso con la divulgación de temas disciplinares contemporáneos, la manera en que el ejercicio profesional se acerca a la construcción de ciudad promueve el debate sobre la formación y la práctica de la arquitectura. Por esta razón, reconocer las dinámicas que la arquitectura y el urbanismo presentan en la modificación de ciudades contemporáneas es uno de los temas permanentes con los que se construye el enfoque del presente medio de divulgación.

En la búsqueda de fomentar la responsabilidad que se aborda desde la disciplina para la construcción de ciudad, es necesario evidenciar que aunque los esfuerzos teóricos de las academias e instituciones gubernamentales fomentan la construcción de ciudades más sostenibles, las urbes latinoamericanas en desarrollo presentan realidades más complejas que implican una gran distancia entre los enfoques teóricos y la realidad.

Por esto, se considera necesario destacar el acercamiento que varios grupos de arquitectos hacen a las zonas periféricas de las ciudades latinoamericanas, los cuales derivan en obras construidas y reconocidas tanto por la comunidad como por entes disciplinares y gubernamentales que premian y reconocen estas prácticas como ejemplos exitosos de la labor social de la arquitectura, promoviendo la discusión acerca de la validez de dichas prácticas dentro de los saberes disciplinares.

Así, el crecimiento de las zonas periféricas y la asidua incidencia de los arquitectos en procesos de intervención de estos territorios con proyectos de pequeña escala, como parte de la construcción de territorio y comunidad, interesan a 
la sección Cultura y Espacio Urbano, por cuanto permiten descifrar actitudes tanto de los profesionales como de las comunidades en función de la consolidación de proceso sociales y culturales que se manifiestan en la ciudad.

\section{Crecimiento de las periferias latinoamericanas sin planeación ni arquitectos}

En el contexto latinoamericano contemporáneo, entendido como el escenario de políticas neoliberales y la exacerbación de las diferencias sociales y de la desigual distribución de las riquezas, el crecimiento desmedido de las ciudades (Pradilla, 2014; Janoschka, 2012) ha consolidado áreas de expansión que albergan desarrollos urbanos carentes de planificación y no consideran la participación de profesionales o técnicos que asesoren o regulen dichos asentamientos.

El crecimiento de estas zonas periféricas es uno de los problemas álgidos de la configuración de la ciudad, donde se requieren reflexiones e intervenciones que promuevan la consolidación de territorios sostenibles, equitativos y eficientes para las comunidades que allí habitan.

La situación es generalizada en las ciudades latinoamericanas, a tal punto que la Organización de las Naciones Unidas (ONU), en su informe mundial sobre las ciudades: World Cities Report. Urbanization and Development, Emerging Futures (2016), asume que uno de los problemas más apremiantes de siglo XXI es el crecimiento descontrolado de las periferias, sumado al aumento de la población vulnerable y los impactos de este crecimiento en el clima.

Por otro lado, este crecimiento aumenta las cifras de la población urbana en la región, por cuanto la Comisión Económica para América Latina y el Caribe (Cepal) (2017) considera que el 79,5\% de la población habita en las ciudades, cifra que va en aumento. Así, el reto de la planificación está en mejorar la calidad de vida de los asentamientos periféricos, a fin de disminuir las desigualdades y fomentar una equidad territorial que reconozca las nuevas dinámicas urbanas.

En este orden de ideas, los documentos preparatorios para el congreso sobre Hábitat, llevado a cabo en 2016 en la ciudad de Quito (Hábitat III), plantean una necesidad en el tratamiento inclusivo para el desarrollo de estos territorios, y permite que se involucre a la comunidad y otros actores dentro de la definición y las actuaciones allí planteadas. Es así como el documento de políticas sobre el derecho a la ciudad señala que:

El logro de una agenda urbana incluyente requiere la participación activa de los agentes fundamentales, como los gobiernos centrales y locales; los círculos académicos; las organizaciones de la sociedad civil; el sector privado; las microempresas y las empresas pequeñas y medianas; el sector no empresarial informal; y los movimientos sociales, entre otros, a fin de transformar las prioridades normativas existentes en medidas concretas y sostenibles. (ONU-Habitat, 2016b, p. 2)

Asimismo, se basa en acciones que propendan por la participación de múltiples actores en los procesos de planificación y gestión de las ciudades como estrategia para abarcar la mayor cantidad de aspectos y visiones sobre el habitar:

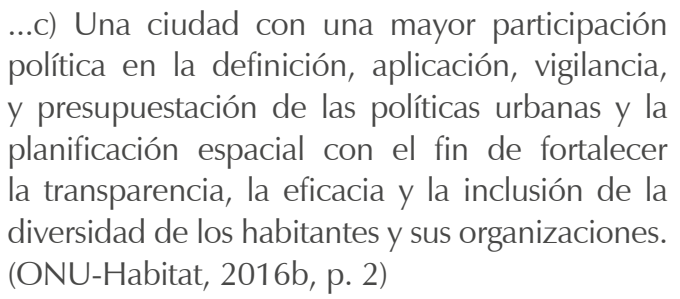

El concepto del derecho a la ciudad acuñado por Lefebvre (1967) y desarrollado por Harvey (2013) en las últimas décadas, apoya los procesos mediante los cuales el ciudadano ejerce el poder de diseñar la ciudad que habita a través de las organizaciones sociales y colectivas, empoderando a las comunidades vulnerables en la construcción de los espacios urbanos que ocupa. Así se reivindican las prácticas donde las comunidades asumen la gestión y construcción de sus espacios y reconocen la escasa participación del apoyo técnico e institucional en dichos procesos.

Sin embargo, actualmente las prácticas de la intervención de la arquitectura y el urbanismo en el desarrollo de las ciudades se ven normalizadas por políticas de ordenamiento territorial que limitan las intervenciones en las zonas periféricas, lo que produce efectos nocivos como la invisibilidad de su desarrollo y sus comunidades, y su escasa participación en los procesos de construcción de la ciudad. Según Isabel Arteaga (2005), la carencia de los territorios periféricos, en cuanto a infraestructura y servicios, obliga a tener una estrecha relación de dependencia con el centro de la ciudad, lo que dificulta las condiciones de vida digna en estos territorios.

\section{Iniciativas emergentes desde la arquitectura y el urbanismo}

Al revisar las prácticas urbanas del siglo XXI, soluciones como el denominado urbanismo táctico (Steffens, 2014), o el urbanismo participativo que menciona Hernández-Araque (2016), utilizan los procesos de crowdsourcing (Brabham, 2013) para considerar la importancia de la participación comunitaria en la construcción de ciudad. Asimismo, conceptos como la acupuntura urbana (Lerner, 2005) destacan aquellas pequeñas acciones que se realizan en diversos territorios y que generan impactos significativos en los sectores y las comunidades.

Son estas realidades, presentes en la práctica de la arquitectura y el urbanismo del siglo XXI en las ciudades latinoamericanas, las que suscitan esta reflexión que se apoya en la pregunta: ¿cómo participan los arquitectos de la construcción de la ciudad incidiendo en las transformaciones 
de los sectores periféricos?, y llevan a considerar los alcances de la enseñanza en la formación de profesionales que incidan en estos procesos y territorios.

Desde los ámbitos de la formación y la investigación local, estas dinámicas de la arquitectura con trabajo comunitario se pueden validar por intermedio de la investigación-creación ${ }^{1}$, entendida como el proceso y el resultado de experiencias que, construidas con la comunidad, dan cuenta de las aproximaciones sensibles que se manifiestan en un obra tangible, que pueden o no ser funcionales y que plantean una relación con la comunidad y la cultura de la que surgen.

\section{La palabra creación hace referencia tanto al pro- ceso, como al resultado de la actividad creativa consistente en explorar diferentes experiencias y sensibilidades a través del juego de formas y materiales que pueden tener o no una funciona- lidad más allá de su valor estético. Los resultados de creación, aunque se plasman en artefactos estéticos, comprenden también las dinámicas entre la obra, las personas que la usan o con- templan y la cultura en la que esta relación está inmersa. En este sentido, el aporte de la creación como generación de conocimiento no radica en el artefacto, sino en la experiencia que este genera y propicia. Por estas razones, en este tipo de actividades creativas el conoci- miento nuevo normalmente es inseparable de su divulgación o publicación y de su apropia- ción social. (Colciencias, 2017, p. 167)}

También, aunque las prácticas de la arquitectura en comunidades vulnerables no son recientes, las bienales de arquitectura, los encuentros académicos o eventos gubernamentales aceptan y celebran estas experiencias a través de la apertura de espacios de discusión académica y disciplinar donde se reconoce el esfuerzo, la labor y el impacto que tienen estos planteamientos dentro de las comunidades, considerando la importancia de la responsabilidad del arquitecto en la construcción de ciudad y sociedad, a través de la aplicación de su oficio.

Una muestra de estos espacios de discusión fue la realización del simposio Medios Informales 2019: Prácticas alternativas en América Latina, organizado por Harvard Graduate School of Design (Figura 1), donde se reunieron quince profesionales de la arquitectura que por sus acciones se relacionan con la comunidad. En el encuentro compartieron saberes acerca de la gestión social, el trabajo comunitario, el trabajo interdisciplinario y nuevas alternativas que conjugan esfuerzos participativos y solidarios de gran impacto social, en comunidades vulnerables.

La consideración de este tipo de trabajos y de la manera en que los arquitectos están impactando positivamente sobre los territorios peri-

1 Categoría de clasificación para los productos de investigación, determinada en el modelo de medición de Colciencias en el año 2017, para permitir la inclusión de producción artística de los académicos y de las facultades de artes de las universidades colombianas. féricos es el tema central de esta reflexión, que busca evidenciar el crecimiento de este interés por parte los profesionales y la manifiesta responsabilidad que se tiene, desde esta labor, para la transformación de los territorios, los modos de habitar y el mejoramiento de la calidad de vida de las personas.

\section{Metodología}

La reflexión presentada surge de la revisión de los proyectos y las acciones implementadas por los convocados al simposio Medios Informales 2019: Prácticas alternativas en América Latina, que consolidó un estado del arte de intervenciones con características similares construidas en la región, con el fin de entender las actuaciones de diversos grupos de arquitectos durante los últimos veinte años, en las zonas periféricas de América Latina.

De manera comparativa se revisaron los postulados de los arquitectos, sus intereses y las memorias de los proyectos, a fin de consolidar una lectura que permitió evidenciar la tendencia de estas prácticas en el siglo XXI. Así, al revisar la información recuperada de las páginas de internet de los arquitectos y a partir del rastreo de diversas publicaciones acerca de prácticas emergentes y reconocimientos a los arquitectos latinoamericanos, se advierte que existen procesos comunes en las intervenciones y prácticas en las periferias latinoamericanas.

El rastreo de la información destacó las declaraciones de los arquitectos y las consideraciones acotadas frente a los proyectos de carácter participativo en zonas periféricas. Se organizó de manera comparativa, destacando los datos que se muestran en la Figura 2, para equiparar los proyectos y poder determinar similitudes y diferencias a la hora de intervenir, e incluso para determinar el carácter de la participación de los distintos entes relacionados durante el proceso.

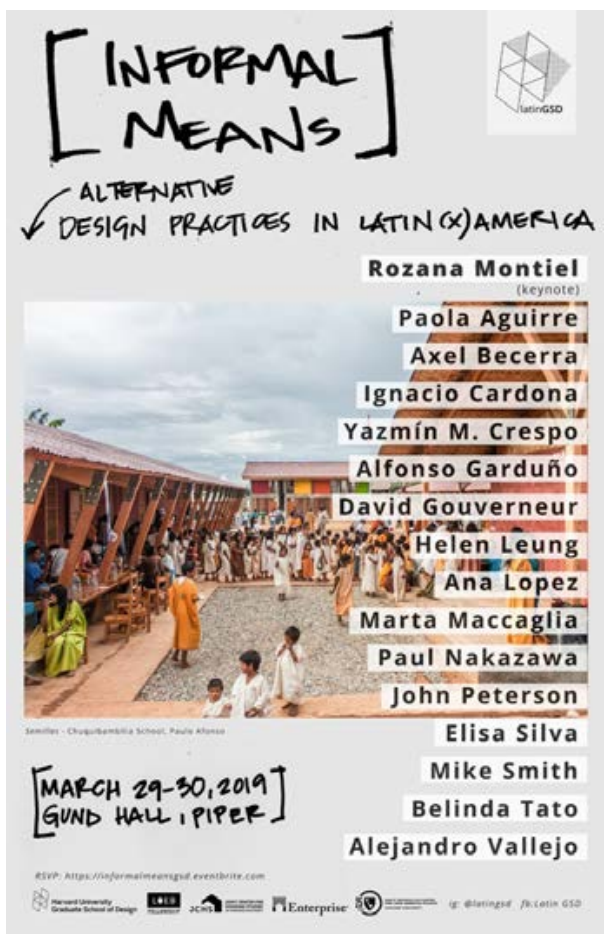

\& Figura 1. Simposio Medios Informales 2019: Prácticas alternativas en América Latina Fuente: Informal Means Alternative Design practices in Latin America (2019). 


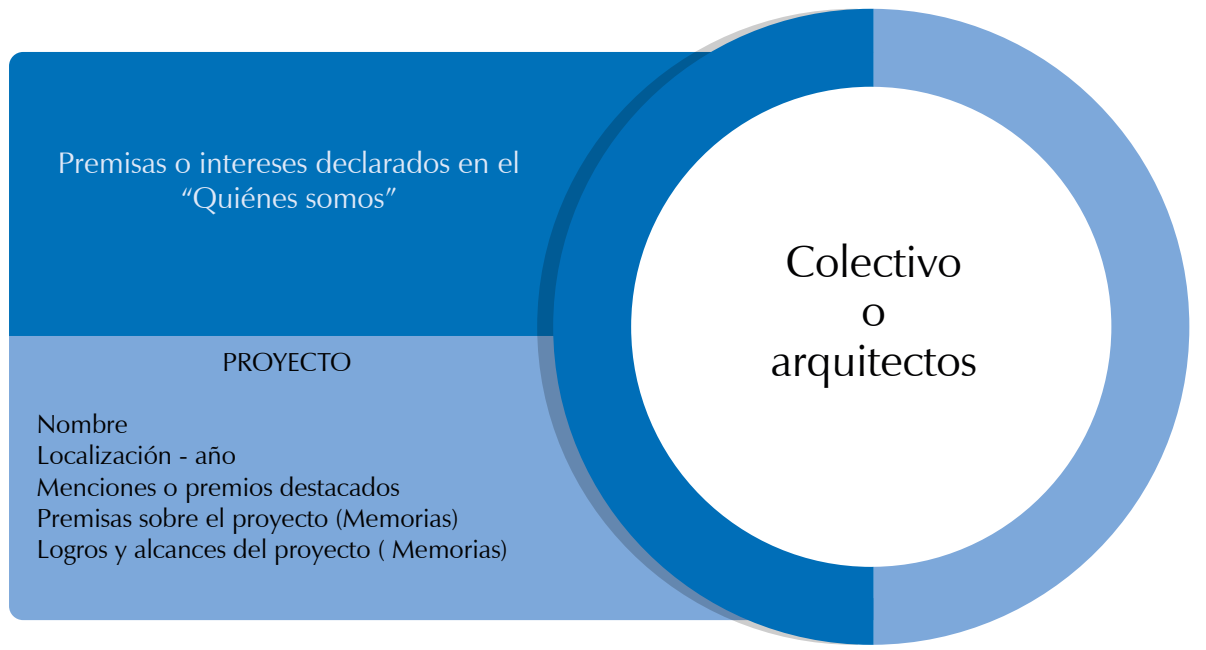

(A) Figura 2. Esquema de revisión de la información Fuente: elaboración propia, 2019. CC BY

También se realizó una interpretación sobre los actores y la intensidad de participación de estos en los proyectos analizados, y se identificaron los premios recibidos por algunos de los proyectos y equipos. Asimismo, se reconocieron las publicaciones realizadas que buscan incentivar la práctica en estos escenarios y que por medio de procesos colectivos y participativos logran generar estrategias para la construcción de ciudades más equitativas.

\section{Resultados}

Las distintas intervenciones que se evidencian en la revisión de los convocados al seminario configuran una postura frente al impacto de la arquitectura, no solo en el desarrollo de la ciudad, sino también en la incidencia sobre la construcción de sociedad. La mayoría de los participantes está aportando de manera tangible a la construcción de territorio y a la consolidación de redes comunitarias.

En la actualidad, el desarrollo del oficio de la arquitectura presenta diversas facetas entre las que se encuentra la vinculada con los procesos tradicionales del oficio, y otras que de manera paralela amplían la perspectiva para el reconocimiento de prácticas alternativas que transforman las lógicas del tradicional proceso cliente-arquitecto e incorporan experiencias colaborativas entre profesionales, oficinas y otros actores que se convocan y apoyan a través de las redes sociales y otros medios contemporáneos, tal como lo afirma Llano Loyola:

La disciplina trabaja hoy en día a modo de un dispositivo abierto que contempla una disposición, una lógica, una decisión bajo un sistema operativo que analiza experiencias y las cartografía en multicapas Ilenos-vacíos-enlaces. Estudiar arquitectura hoy -teórica y materialmente- alude a interconexión por epistemes a-escalares, es decir, por una interdisciplinariedad que busca en las metodologías impactar en el sujeto bajo transferencias de conocimiento ya no por acumulaciones sino por construcciones determinadas de herramientas y competencias afines. (2017, p. 372)

La constante colaboración entre grupos para diversos alcances de la disciplina ha llevado a los arquitectos a trascender la idea de oficina de arquitectura tradicional con un arquitecto líder y otros colaboradores, para configurar colectivos con intereses comunes, pero con conocimientos diversos, los cuales apuntan a un fin común, que puede o no derivar en un ejercicio arquitectónico tangible. Eso no quiere decir que no sea el objetivo del arquitecto, pero ya no es el único fin.

De esta manera, y a través del reconocimiento de otros actores y agentes que inciden en el territorio y en la construcción de ciudad, los arquitectos se han acercado a las comunidades, a sus líderes y, junto con ellos, han propiciado procesos participativos que derivan en acciones físicas en los territorios, que se reconocen por el impacto de su presencia y apropiación por parte de los habitantes de las zonas en las que se construyen.

Esta participación recurrente de los arquitectos en las intervenciones de los territorios periféricos destaca las lógicas de los procesos de ocupación propios del escenario que, en su mayoría, no obedecen las formas tradicionales de construir ciudad, lo que valida los procesos de autoconstrucción, ayuda mutua, esfuerzo propio y gestión colectiva que se identifican en la teoría del derecho a la ciudad, aplicado a comunidades y territorios vulnerables, hecho que evidencia los deficientes procesos de una planificación urbana incluyente, participativa y eficiente.

De esta manera, se entiende que la arquitectura participativa es una estrategia de acción con la cual se puede acercar el oficio propio del técnico a ámbitos más humanos, donde se consideran las necesidades de las comunidades más allá del hecho práctico, y se valoran los procesos de integración, colaboración y construcción de comunidad, como los pilares sobre los que descansará cualquier intervención en el territorio.

El término arquitectura participativa está en auge dentro de las prácticas y la teoría de la arquitectura reciente. Esta situación permite reflexionar acerca de la importancia de la comprensión y el abordaje de esta estrategia dentro del desarrollo del oficio y la construcción de ciudad.

Según lo menciona William García (2012), la arquitectura participativa se concreta en la relación entre el arquitecto y la comunidad. Bajo estos principios se reconocen tres modelos de asociación: el arquitecto dirigente, el arquitecto subalterno y el arquitecto intérprete. En cada una de ellas, las relaciones entre el arquitecto y la comunidad generan prácticas diversas, cuyos resultados varían en su propuesta frente al territorio, la comunidad y las necesidades.

En ese orden de ideas, dentro de los procesos revisados se identifica la actitud del intérprete como elemento transversal a los proyectos desarrollados por los arquitectos. También se destaca la presencia de otros actores entre los que se encuentra la comunidad e incluso los estados locales, situación que también supone un rol distinto desde el arquitecto para establecer diálogos y acuerdos entre las diversas instancias. 
Las actuaciones de los arquitectos intérpretes frente a la comunidad se establecen mediante acercamientos a través de talleres de reconocimiento, trabajo y discusión sobre las diferentes etapas del proyecto, basadas en las teorías del diseño participativo. Es en estos encuentros donde se identifican las prácticas, los deseos, los intereses y las necesidades de la comunidad. Aquí, y a través del oficio, se generan alternativas para la manifestación física de estos aspectos en una intervención tangible en el territorio, que será de nuevo retroalimentada y discutida con la comunidad, hasta llegar a su construcción, de ser posible.

Participatory Design projects are always driven by ongoing and systematic reflection on how to involve users as full partners in design and how this involvement can unfold throughout the design process. The basic motivation remains democratic and emancipatory: Active participation needs to define Participatory Design because if we are to design the futures we wish to live, then those whose futures are affected must actively participate in the design process ${ }^{2}$. (Robertson y Simonsen, 2012, p. 5)

Por otro lado, la inmersión del arquitecto en los procesos participativos con las comunidades $y$, en ocasiones, con las instituciones gubernamentales, fomenta el rol del intérprete, por cuanto son los arquitectos quienes apoyan los procesos de gestión y median entre los intereses de la comunidad y las situaciones propias de los procesos institucionales, mediación a través de la cual a veces logran el apoyo material, técnico, burocrático, entre otros, de estos procesos originados en el empoderamiento ciudadano.

De esta manera, y como lo menciona Llano Loyola (2017), el trabajo en los territorios periféricos actúa como un laboratorio, donde el rol del arquitecto se alinea con los procesos de gestión, pero a la vez le permite desarrollar el trabajo material de la obra, desde esa noción de arquitecturas colectivas.

Además de la comunidad y de la institución, los arquitectos, en los procesos de intervención en las zonas periféricas, coinciden con otros profesionales estableciendo escenarios de diálogo interdisciplinar, lo que aboga por un rol más conciliador y receptivo a la comprensión holística del escenario.

La interdisciplinariedad lineal tipo 1 se da cuando varias disciplinas abordan un mismo problema $u$ objeto de estudio y cada una aporta desde su saber elementos para una mejor comprensión. Pero ninguna sufre cambios o modificaciones determina-

2 "Los proyectos de diseño participativo siempre están impulsados por una reflexión continua y sistemática sobre la manera de involucrar a los usuarios como socios completos en el diseño, y cómo esta participación puede desarrollarse a lo largo del proceso. La motivación básica sigue siendo democrática y emancipadora: la participación activa debe definir el Diseño Participativo, porque si queremos diseñar los futuros que deseamos vivir, entonces aquellos que se ven afectados deben participar activamente en el proceso de diseño" (traducción propia). bles. El problema es el centro de la acción y su impacto está determinado por la pertinencia de la interacción de las disciplinas en aportar nuevas soluciones. (Peñuela, 2005, p. 75)

Aquí el arquitecto ofrece su conocimiento y capacidad de materialización de la intervención como un pretexto para propiciar un laboratorio de búsquedas en los ámbitos sociales, antropológicos, políticos, económicos, ambientales, entre otros. Se podría decir que el edificio se convierte en un pretexto para lograr procesos de transformación social de los sectores o de las comunidades vulnerables. Es así como los procesos de gestión adquieren más vigor que los mismos hechos físicos.

\footnotetext{
Entre este tipo de arquitectos la novedad se identifica con la incorporación al repertorio de herramientas que provienen de la sociología, la política, la antropología, la economía o la ecología. Los nuevos arquitectos amplían y trascienden los límites de la multidisciplinariedad profesional planteados en la agenda moderna de arquitectos iberoamericanos. Para ellos, la arquitectura no son objetos, sino sistemas de trabajo, y manejan temas como el bajo presupuesto, las necesidades básicas, las preocupaciones políticas y sociales, la movilidad, la temporalidad y la subversión conceptual. (Vial, 2008)
}

Otra de las similitudes de los arquitectos estudiados radica en sus declaraciones acerca de las responsabilidades sociales de la arquitectura, por encima de las constructivas o técnicas. En los discursos, los profesionales resaltan el interés por el trabajo colectivo entre arquitectos, pero también la necesidad de incluir diversos profesionales que apoyen el proceso social. Conceptos como la equidad social, la transformación y creación de comunidades, el diseño colaborativo y participativo, la investigación aplicada, el diálogo entre arquitectura y ciudad, entre otros, reúnen sus propuestas en una nueva visión de la disciplina.

Al revisar los proyectos es claro tanto el ejercicio participativo como el interdisciplinar. Los arquitectos, en su labor de intérpretes, desarroIlan procesos con los habitantes y con la institución con el fin de consolidar las intervenciones y proveer a las comunidades de las soluciones espaciales que les permitan fortalecer las transformaciones territoriales, sociales y culturales de sus barrios.

Es así como, dependiendo del proyecto, la comunidad y la relación con la institución se pueden establecer diversas intensidades de participación de cada uno de los distintos actores entre los que se encuentran la comunidad, los arquitectos, las instituciones privadas, los gobiernos locales y otros profesionales. En la Figura 3 se evidencian los actores rescatados de los proyectos participativos revisados que, en el marco de lo ideal, trabajan juntos y de manera equilibrada para dar cuerpo al proceso y a las materializaciones.

Sin embargo, cada intervención es distinta y plantea diversidad de relaciones y de intensidades 


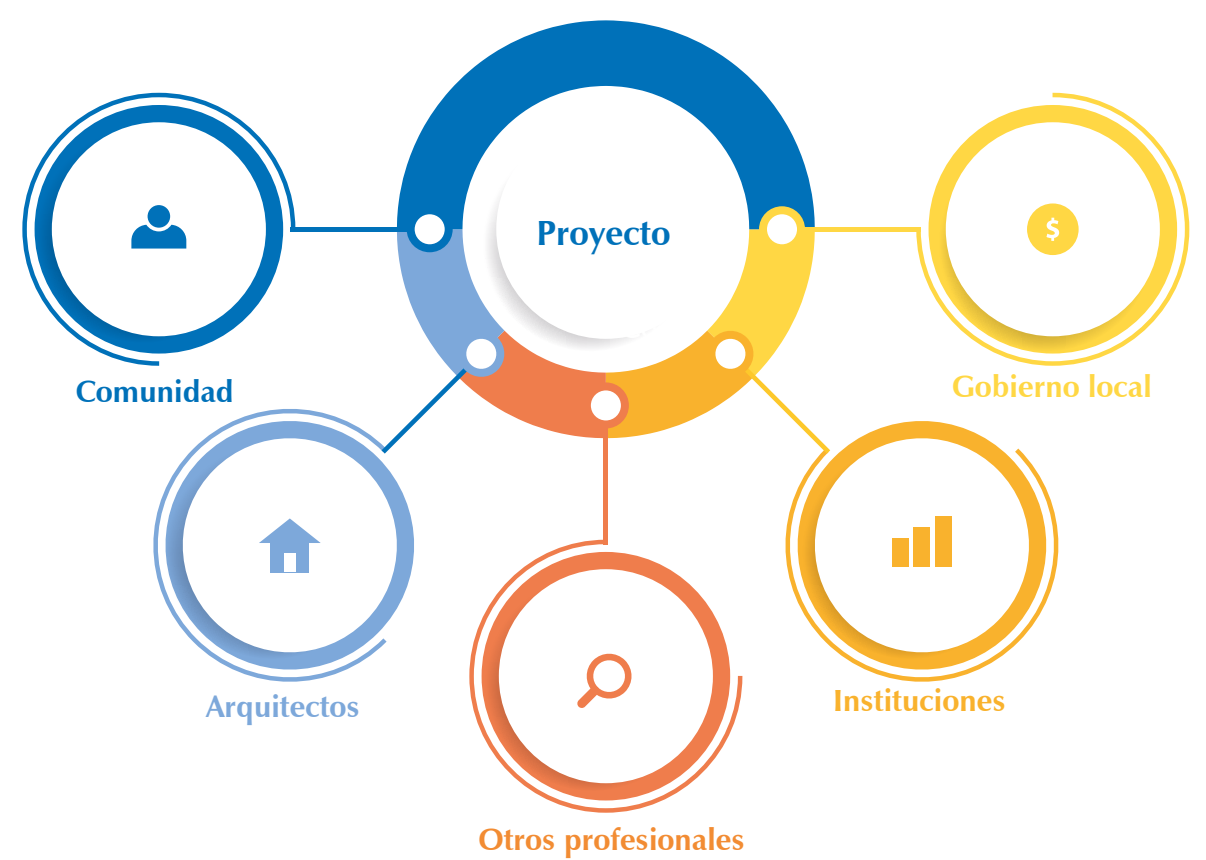

(A) Figura 3. Actores del proceso participativo. Ideales Fuente: elaboración propia, 2019. CC BY

de participación. Para dar cuenta de ello se exponen tres casos extraídos del análisis de información.

En primera instancia, en el proyecto del equipo de Al Borde arquitectos, Escuela Rural Nueva Esperanza, en Manabí (Ecuador), donde las necesidades de la comunidad son expuestas a los arquitectos en diversos encuentros, son ellos quienes las interpretan y llegan a la conclusión de establecer un espacio abierto con una función inicial de escuela, pero que sirva para múltiples propósitos. Por otro lado, la carencia de un auspicio institucional obliga a considerar la construcción con materiales propios de la zona y a utilizar la mano de obra local, integrando aún más a la comunidad al proceso a fin de limitar los gastos.

En este caso, el esquema de intensidades de participación se muestra en la Figura 4, donde comunidad y arquitectos establecen las relaciones y actúan paralelamente en la concreción del ejercicio.

En otros casos, la presencia del gobierno local hace parte del proceso que se lleva a cabo con el proyecto. El Multiprogram Ship, de Alejandro Haiek y Lab. Pro. Fab (Figura 5), en la ciudad de Caracas (Venezuela), se consolida gracias a la gestión de los consejos comunitarios ${ }^{3}$. A través de la organización comunitaria y la autogestión se configuran los procesos de diseño, discusión y concreción, donde el arquitecto actúa como intérprete de las solicitudes de la comunidad con talleres participativos. Con los recursos públicos se logra dar pie a la construcción y formalizar el funcionamiento del espacio.

El tercer caso es el proceso liderado por el Colectivo Pico de Venezuela, quienes a través

3 Estructuras de administración territoriales avaladas por la Constitución venezolana, que establecen formas de gobernabilidad y permiten el recurso legal para la canalización de los recursos públicos a partir de la vía democrática. de la iniciativa "Espacios de paz" convocaron a otros arquitectos de diversas nacionalidades a trabajar en cinco zonas periféricas de la ciudad de Caracas. Allí se realizaron obras de readecuación, rehabilitación, recuperación, entre otras, de espacios subutilizados, para convertirlos en espacios públicos de activación comunitaria. El proyecto Núcleo Cultural la Ye, del Colectivo Pico (Figura 6), plantea la interacción con varios agentes entre los que se encuentran ellos a la cabeza del proceso, la comunidad, el gobierno local, la empresa Petróleos de Venezuela S.A. (PDVSA) y otros profesionales que apoyaron los talleres logísticos a través del arte urbano.

Estos análisis a las intensidades de participación de los diferentes actores de los procesos participativos ponen de manifiesto la necesidad de incluir al arquitecto dentro de escenarios más abiertos y dinámicos, en donde el diálogo es la herramienta para consolidar procesos y proyectos que tengan un verdadero impacto en las comunidades y en los territorios. Asimismo, plantea la idea de que el rol de gobernanza, gestión y empoderamiento recaiga en la comunidad y se apoye en los demás entes participantes.

Como parte del análisis se puede establecer que varios proyectos fueron reconocidos en bienales y recibieron premios en categorías calificadas como arquitectura emergente o arquitectura social. En este orden de ideas, se destacan los premios del grupo Hábitat sin Fronteras, que fue premiado en la Bienal de Arquitectura de Quito en el año 2012, con el primer premio en la categoría Hábitat social y desarrollo, con su proyecto Preescolar Casa del Caracol, en Panamá, y también con los Premios Arquitectura Diáspora Colombiana $^{4}$ en el año 2012, como proyecto de arquitectura colaborativa.

También se resalta el caso de los arquitectos Alejandro Vallejo y Michael Smith, ganadores del Gran Premio Bienal y del Premio Bienal Nacional -Bienal Internacional de Arquitectura Costa Rica 2016-, con su proyecto La cueva de Luz, realizado con la comunidad de La Carpio, zona periférica de la ciudad de San José en Costa Rica.

El Grupo Talca, de Chile, fue ganador de diversos concursos como el Yap Constructo ${ }^{5}$ (The Young Architects Program) en el año 2014. En el año 2015 ganaron el premio del International Committee of Architectural Critics (CICA) para jóvenes arquitectos. Para 2016, fueron parte de los grupos invitados en selección oficial para participar en la Bienal de Arquitectura en Venecia.

En el ámbito local, el colectivo Arquitectura Expandida recibió premios que se relacionan con sus procesos como parte del impulso a la

4 Premios Arquitectura Diáspora Colombiana, premio bienal que reconoce los proyectos de arquitectos colombianos en el exterior.

5 Concurso de colaboración anual entre el Museo de Arte Moderno y el MoMa PS1, cuyo fin es "fomentar la investigación en diseño innovador y promover el talento emergente". 


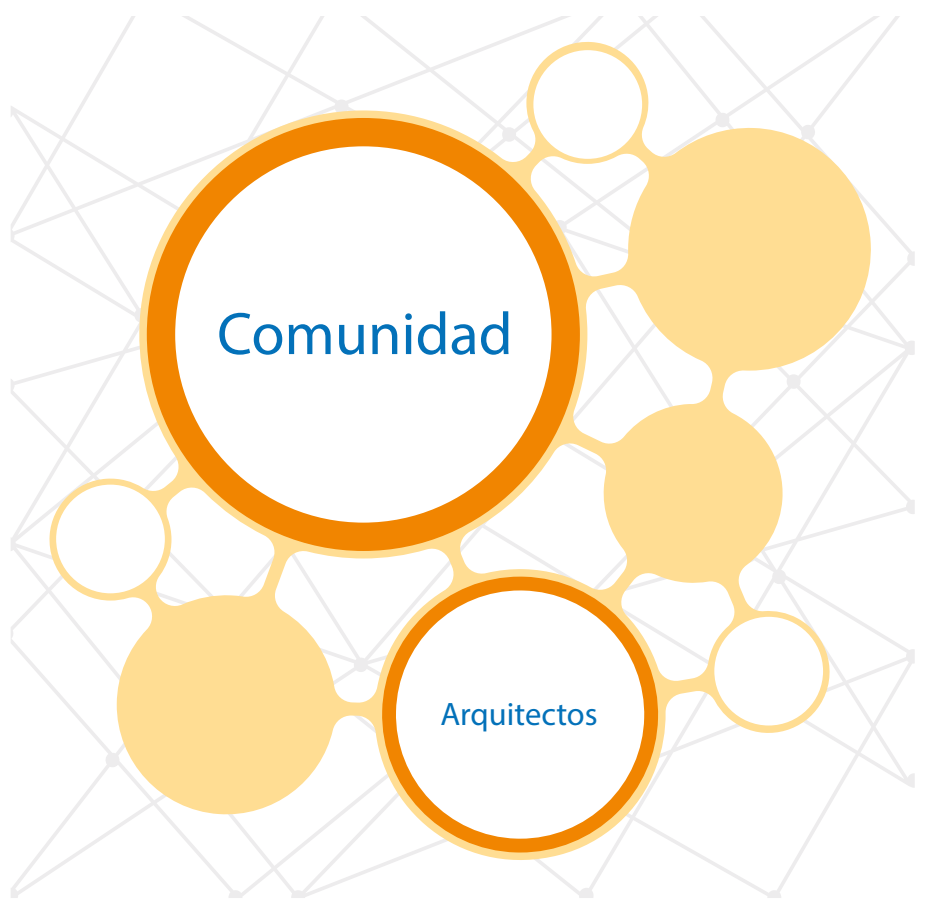

construcción de ciudad; en el 2012 la Secretaría Distrital de Cultura, Recreación y Deporte de la Alcaldía Mayor de Bogotá los reconoció con el premio "Amor por Bogotá: experiencias exitosas que promovieron espacios interculturales y construcción de cultura democrática".

Para el 2013, la Fundación Corona, Fundación Plan, Casa Editorial El Tiempo y la Fundación Bolívar-Davivienda les otorgaron el segundo lugar en el "Premio cívico-Por una ciudad mejor" ${ }^{\prime}$, al reconocer los "Encuentros de arquitectura expandida" como laboratorio de innovación social.

Aunque otros proyectos también han sido reconocidos, este tipo de afirmaciones en el ámbito disciplinar fortalece el interés de dar a la arquitectura un rol más cooperativo en la construcción de las ciudades, donde la arquitectura es el pretexto para la construcción de comunidad y la mejora en la calidad de vida de innumerables habitantes en condición de vulnerabilidad.

Además, de esta manera de divulgación de las arquitecturas colectivas es importante destacar el ejercicio teórico que realizan los colectivos de arquitectos, a través de la producción de estudios y publicaciones que se derivan de los procesos de gestión que se llevan a cabo en las periferias. Es aquí donde los gestores avalan la imperante necesidad de tener en cuenta a las comunidades en las acciones de diseño y los resultados de estos.

Estas publicaciones son referentes académicos y técnicos a la hora de considerar transformaciones en los procesos de gestión con comunidades vulnerables en sectores conflictivos de las periferias, y son altamente valorados como estudios de caso para replicar.

6 Reconocimiento anual a iniciativas sociales que trabajen de manera colectiva para mejorar el hábitat de comunidades.

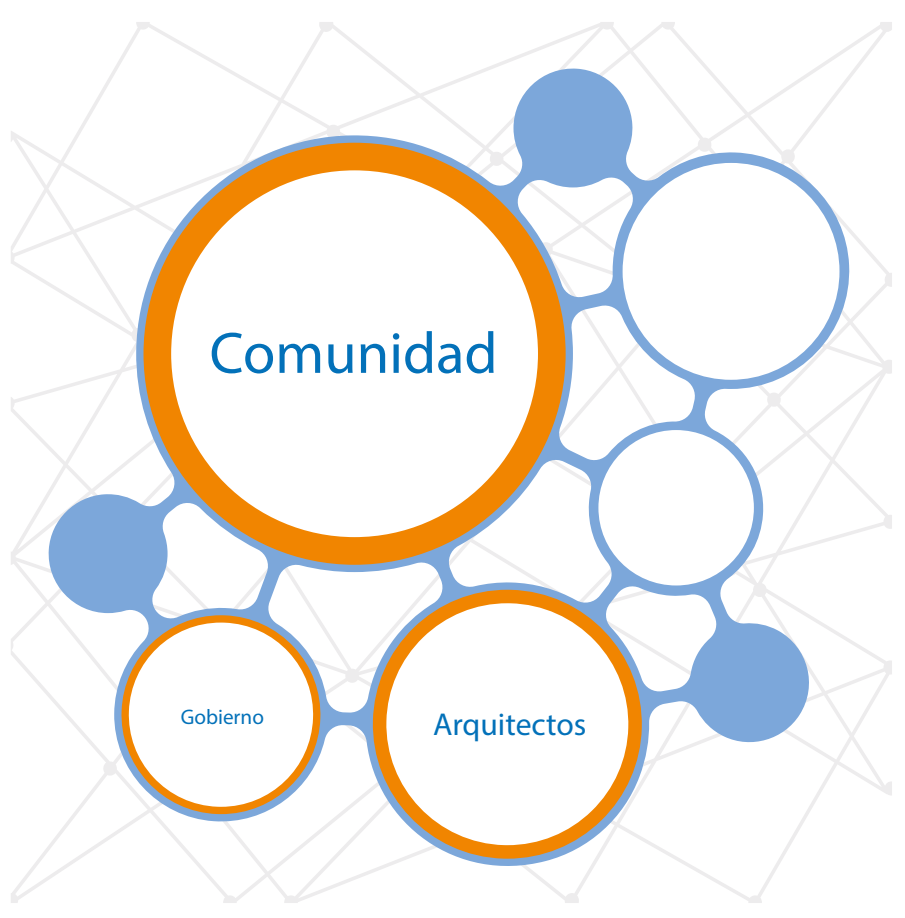

(A) Figura 4. Diagrama de actores e intensidades Escuela Nueva Esperanza Fuente: elaboración propia, 2019. CC BY
(A) (A) Figura 5. Esquema de actores e intensidades. Proyecto Multiprogram Ship Fuente: elaboración propia, 2019. CC BY

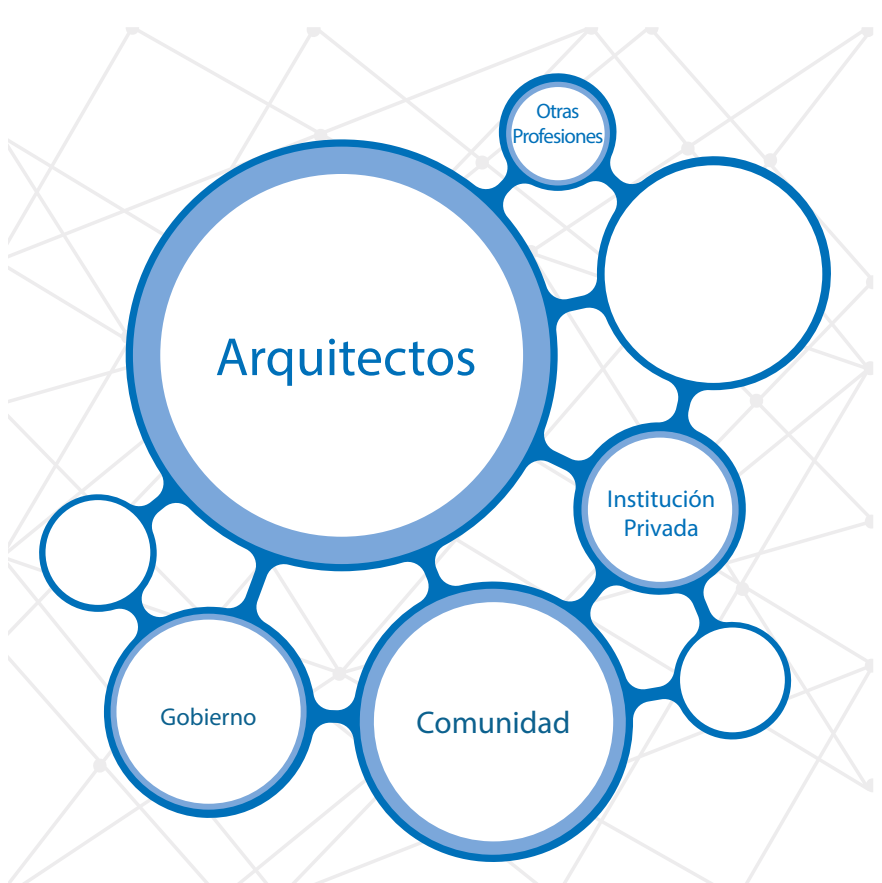

(A) Figura 6. Esquema de actores e intensidades. Proyecto Núcleo Cultural la Ye

Tal es el caso de los arquitectos del colectivo Fuente: elaboración propia, 2019. CC BY
Arquitectura Expandida, con la publicación Los comunes urbano-rurales del Alto Fucha. En riesgo (Figura 7), donde evidencian procesos que se realizaron en la cuenca del río Fucha en el sur oriente de la ciudad de Bogotá (Colombia) para la ocupación de espacios urbanos que quedaron baldíos por los procesos de gestión de riesgo de la zona periférica de ladera del río Fucha.

Otro documento valioso para el tema de las intervenciones en zonas periféricas y con comunidades vulnerables es el libro Diseño de nuevos 


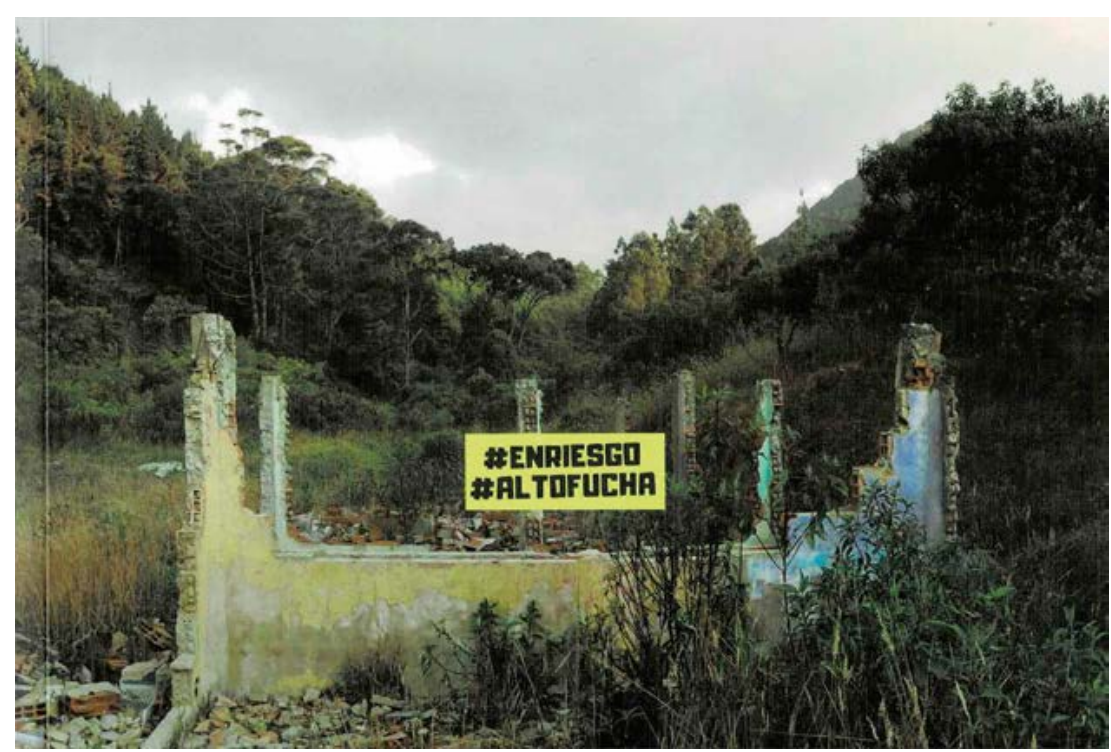

(A) Figura 7. Portada del libro Los comunes urbano-rurales del Alto Fucha. En riesgo Fuente: Arquitectura Expandida - Colectivo Huertopía (2019)

? Figura 8. Portada del libro Diseño de nuevos asentamientos informales (2016)

Fuente: Gouverneur (2016).

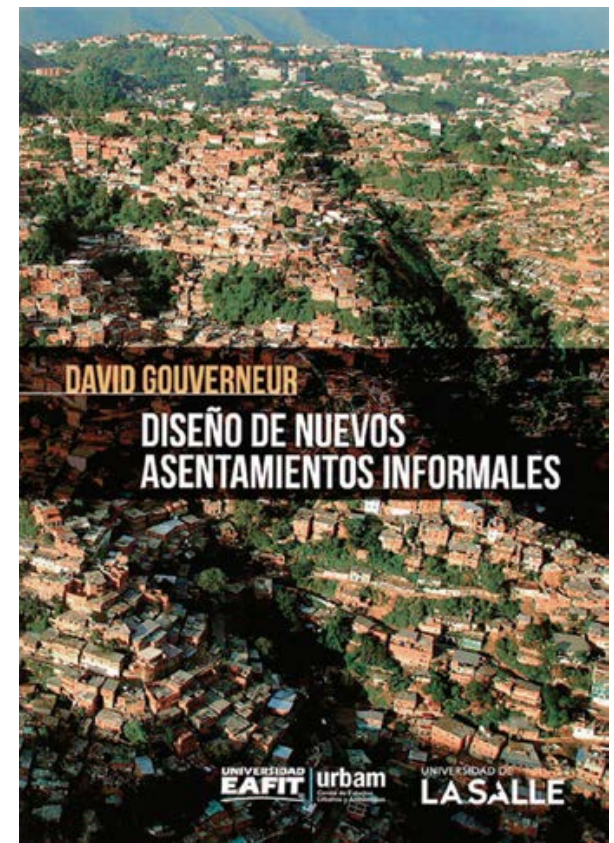

asentamientos informales, escrito por el arquitecto David Gouverneur (2016) (Figura 8), donde el autor muestra la investigación realizada durante 25 años sobre la planeación de los asentamientos informales como resultado de la práctica docente y de la mano del trabajo en el sector público; en esta se hace evidente la necesidad de incorporar estrategias de diseño interdisciplinares que aborden las dinámicas de la construcción de la ciudad contemporánea y, específicamente, en la planeación de los asentamientos informales.

La revisión del panorama de arquitectos y proyectos derivó en una comprensión del fenómeno emergente, identificando las situaciones ya presentadas; asimismo, desde las mismas intenciones de los arquitectos, expresadas en sus discursos y acciones, se intuye el interés de dar una nueva mirada al ejercicio profesional, liberando al arquitecto de la potestad de imponer las soluciones para el habitar desde la disciplina a fin de acercarse más a su rol de ciudadano participante. Como lo menciona Natalia Yunis (2015), frente a la postura propuesta por Haiek acerca del arquitecto: "sustituyendo el rol del arquitecto y urbanista por aquel del ciudadano se puede crear una fuerza poderosa, capaz de replicar experiencias y generar una colaboración integradora".

\section{Discusión}

Frente a los temas expuestos y la manera en que construyen ciudad, la apuesta por la pequeña escala es parte del nuevo discurso de la arquitectura propuesto por las acciones de los arquitectos latinoamericanos en las zonas periféricas.

A través de los ejercicios participativos, donde se incluyen distintos actores con diversas incidencias, se propone que la arquitectura sea el pretexto para abordar situaciones de la planeación territorial y de la comprensión de las dinámicas sociales de las comunidades que ocupan los territorios.

Los arquitectos analizados le apuestan a que las pequeñas acciones que logran consolidar en los escenarios periféricos, además de solucionar un problema puntual de la comunidad, se conviertan en catalizadores de la vida urbana, en centros comunitarios capaces de activar relaciones entre vecinos, actividades para los jóvenes, espacios de representación social e incluso de participación política.

Desde el punto de vista de la transformación territorial, la mayoría de los casos revisados tienen la posibilidad de ser replicables y, en ocasiones, esto se ha logrado, como los proyectos del Colectivo Pico, de Arquitectura Expandida entre Nos Atelier o de Al borde Arquitectos. De procesos iniciales, que se consiguieron en una etapa experimental, hoy se gestionan con otras comunidades y con mayor asertividad nuevos eventos arquitectónicos, que demuestran que las obras tangibles, a pequeña escala, con participación de la comunidad y apoyo institucional, realmente magnifican la calidad de vida de las personas que viven en estos territorios y los visibiliza frente al resto de la ciudad y de la sociedad.

En muchos casos, estos proyectos se insertan dentro de dinámicas de turismo de las ciudades y logran incluir a estos territorios y a sus comunidades dentro de las dinámicas económicas, de las que antes estaban excluidos. Lo anterior aporta al aumento del empleo, la capacitación y la participación de los habitantes en la construcción de una ciudad sostenible y equitativa.

Es aquí donde se identifica el verdadero aporte que hacen estas intervenciones que, por suerte, cada vez son mayores y trascienden a las grandes intervenciones urbanas de renovación o desarrollo de zonas, las cuales excluyen participantes y se enfocan en visiones económicas que no se concretan en cortos plazos y tienen unos costos elevados, que normalmente las hacen inviables. De esta manera, se debe propender por acciones más medidas, viables y con participación de múltiples actores que las diseñarán, gestionarán y mantendrán, para propiciar transformaciones reales. 


\section{Conclusiones}

Las arquitecturas colectivas son una manifestación de la importancia que vuelven a tener los procesos de acercamiento a las comunidades con intereses de aprender y establecer diálogos, antes que de imponer o desconocer las dinámicas propias de los territorios. Se podría decir que el edificio se convierte en un pretexto para lograr procesos de transformación social de los sectores o de las comunidades vulnerables. Es así como adquieren más vigor los procesos de gestión, que los mismos hechos físicos.

Al ser una práctica relativamente reciente, se ha teorizado poco alrededor de las formas en que se desarrollan estos ejercicios, situación que contrasta con el aumento en los reconocimientos, los eventos y el fortalecimiento de las prácticas en el escenario real.

Vale la pena continuar indagando al respecto, ya que estas prácticas trascienden el modelo tradicional del oficio y la disciplina para involucrarse con preocupaciones sociales y políticas, abogando así por el reconocimiento de territorios y comunidades que, de otra manera, seguirían al margen de la intervención estatal y de la inclusión en las dinámicas formales de las ciudades.

Quedan abiertas las propuestas para revisiones más profundas de otras firmas o estudios de arquitectura, diferentes a las aquí referenciadas, para dar cuenta de los diversos intereses que mueven a estos arquitectos a participar de los procesos de construcción de la ciudad periférica, o para encontrar y clasificar sus actuaciones de acuerdo con los aspectos disciplinares, tarea que se está construyendo desde diversos ámbitos.

Reconociendo el panorama es como estas prácticas alternativas de las arquitecturas contemporáneas abrirán nuevos caminos para la comprensión del oficio, la enseñanza, la investigación y el debate sobre la responsabilidad de la arquitectura con la construcción del mundo que habitamos.

\section{Referencias}

Arquitectura Expandida y Colectivo Huertopía (2019). Los Comunes urbano-rurales del Alto Fucha. En riego. Bogotá: Alcaldía de Bogotá, Secretaría de Cultura, Recreación y Deporte. Empresa de Acueducto y Alcantarillado de Bogotá. Recuperado de https:// issuu.com/arquitecturaexpandida/docs/ enriesgo

Arteaga I. (2005, enero-diciembre). De periferia a ciudad consolidada. Estrategias para la transformación de zonas urbanas marginales. Revista Bitácora Urbano Territorial, 9(1), 98-111. Recuperado de https://revistas.unal.edu.co/index.php/bitacora/article/ view/18741

Brabham, D. (2013). Crowdsourcing. Cambridge: The MIT Press Essential Knowledge Series. Recuperado de http://wtf.tw/ref/brabham.pdf

Cepal y UN-Habitat (2017). Informe regional América Latina y el Caribe: ciudades sostenibles con igualdad. Quito: Cepal y UN-Habitat. Recuperado de https://repositorio.cepal.org/bitstream/handle/11362/40658/1/S1601057 es.pdf

Colciencias (2017). Modelo de medición de grupos de investigación. Convocatoria 781. Bogotá: Colciencias. Recuperado de https:// www.colciencias.gov.co/sistemas-informacion/modelo-medicion-grupos

García Ramírez, W. (2012). Arquitectura participativa: las formas de lo esencial. Revista de Arquitectura (Bogotá), 14(1), 4-11. Recuperado de https://editorial.ucatolica.edu.co/ ojsucatolica/revistas_ucatolica/index.php/ RevArq/article/view/721

Gouverneur, D. (2016). Diseño de nuevos asentamientos informales. Medellín: Eafit.
Harvey, D. (2013). Ciudades rebeldes. Del derecho de la ciudad a la revolución urbana (trad. J. Madariaga). Madrid: Akal.

Hernández-Araque,M.(2016). Urbanismo participativo. Construcción social del espacio urbano. Revista de Arquitectura (Bogotá), 18(1), 6-17. http://dx.doi.org/10.14718/RevArq. 2016.18.1.2

Informal Means: Alternative Design Practices in Latin(x) America (2019, marzo). Archdaily. [Poster] Recuperado de https://www.archdaily.co/co/913988/informal-means-alternative-design-practices-in-latin-x-america

Janoschka, M. (2012). Geografías urbanas en la era del neoliberalismo. Una conceptualización de la resistencia local a través de la participación y la ciudadanía urbana. Investigaciones geográficas, 0(76), 118-132. http://dx.doi.org/10.14350/rig.29879

Lefebvre, H. (1967). El derecho a la ciudad. Barcelona: Península.

Lerner, J. (2005). Acupuntura urbana. Barcelona: Institut d'Arquitectura Avançada de Catalunya.

Llano Loyola, J. (2017). Yo como colectivo: grupos de formación arquitectural en la época del capitalismo diy. En P. Moisset (ed.). La experimentación proyectual: Actas VIII Projetar 2017. Buenos Aires: Universidad de Buenos Aires, FADU-Instituto de la Espacialidad Humana y otros.

ONU-Habitat (2016a). Word Cities Report. Urbanization and Development. Emerging Futures. ONU-Hábitat: Recuperado de http://wcr.unhabitat.org/wcr downloads/ world-cities-report-2016-abridged-edition/
ONU-Habitat (2016b). Documento de política 1: derecho a la ciudad y ciudades para todos. Naciones Unidas, Asamblea General. Recuperado de http://habitat3.org/wp-content/ uploads/Policy-Paper-1-Espan\%CC\%83ol. pdf

Peñuela Velásquez, A. (2005, junio). La transdisciplinariedad. Más allá de los conceptos, la dialéctica. Andamios 2(1), 43-77. https:// doi.org/10.29092/uacm.v0i2.492

Pradilla Cobos, E. (2014). La ciudad capitalista en el patrón neoliberal de acumulación en América Latina. Cadernos Metrópole, 31(16), 37-60. http://dx.doi.org/10.1590/22369996.2014-3102

Robertson, T. y Simonsen, J. (2012). Challenges and opportunities in contemporary participatory design. Design Issues, 28(3), 3-9. https://doi.org/10.1162/DESI_a_00157

Steffens, K. (2014). Urbanismo táctico, 3 casos latinoamericanos. Ciudad Emergente. Santiago de Chile: Fundación Ciudad Emergente. Recuperado de https://ciudademergente.org/aprender/urbanismo-tctico-3

Vial, C. (2008, agosto 8). 2G Dossier. Iberoamérica. Arquitectura Emergente. Archdaily. Recuperado de https://www.archdaily.co/ co/02-9308/2g-dossier-iberoamerica-arquitectura-emergente

Yunis N. (2015, octubre 3). Alejandro Haiek: Debemos sustituir el rol del experto por el del ciudadano. Archdaily. Recuperado de https://www. archdaily.co/co/774700/alejandro-haiek-coII-debemos-sustituir-el-rol-del-experto-por-el-del-ciudadano 



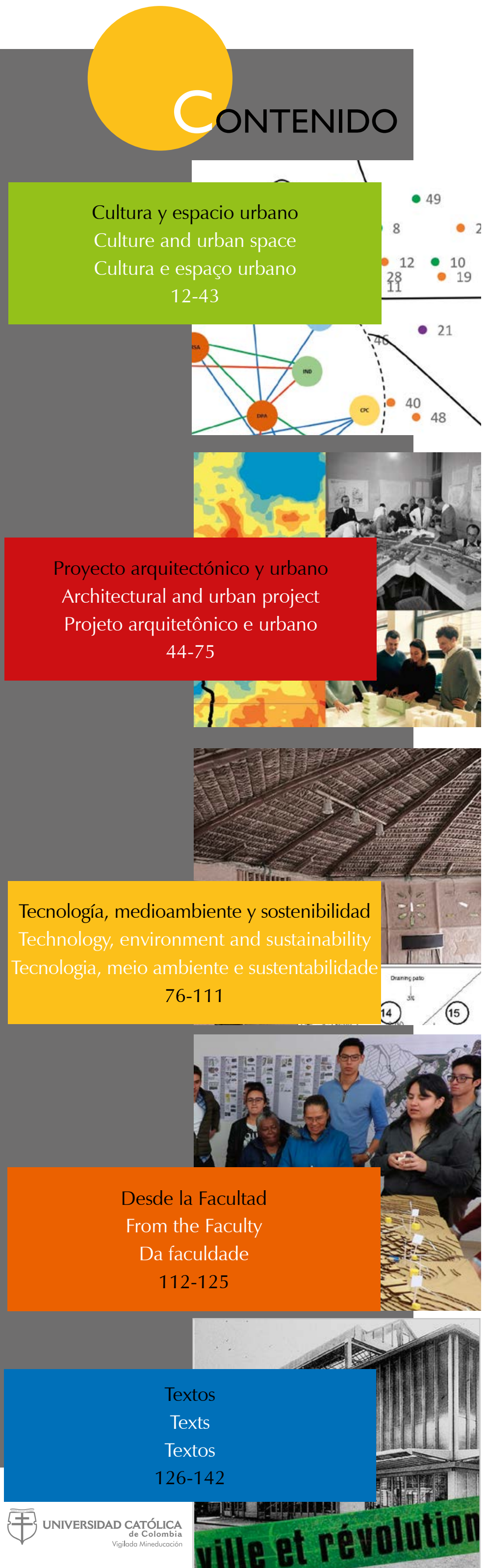

Arquitecturas colectivas y participación como estrategias para la construcción de la ciudad latinoamericana

Myriam Stella Díaz-Osorio

Pág. 3

ES

La caminabilidad en Bogotá: propósitos y condiciones socioespaciales que facilitan y limitan esta experiencia Pablo Páramo

Andrea Burbano

Pág. 12

ES EN

Planificación comunitaria en barrios socialmente

vulnerables. Identificación de los actores sociales en una comunidad

Rafael Alejandro Tavares-Martínez
Jesús Manuel Fitch-Osuna

ES

Pág. 22

Desvanecimiento de la frontera como límite. Imaginario del borde como espacio público físico y virtual

Gabriela Eloísa Muñoz-Torres

Susana Gutiérrez-Luna

Pág. 33

Estudiantes latinoamericanos en el Institut

d'Urbanisme de l'Université de Paris (1923-1941)

$$
\begin{aligned}
& \text { Andrés Ávila-Gómez } \quad \text { Pág. } 44 \\
& \text { ES }
\end{aligned}
$$

Apuntes para el repensamiento de la enseñanza de la Arquitectura. La cuestión epistemológica y la necesidad de una razón ampliada
Juan J. Álvarez-Álvarez
Pág. 57

ES

Equipamientos colectivos: "lugares" de producción de capital social
José Mario Mayorga-Henao
Pág. 68

\section{ES}

Bucle multidisciplinar para la sustentabilidad urbana

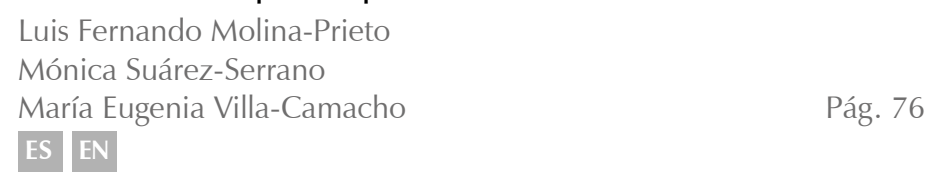

Durabilidad de los materiales naturales de construcción: percepciones de proyectistas, constructores y usuarios en Florianópolis, Brasil

Andrea Salomé Jaramillo-Benavides
Zuleica Maria Patricio-Karnopp
Lisiane Ilha-Librelotto

Pág. 89

\section{ES}

Thermal comfort in buildings for wet processing of coffee

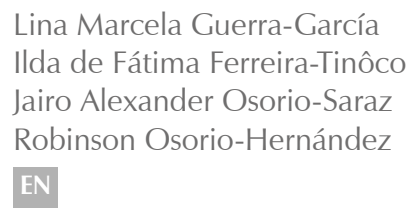

La arquitectura en los barrios: puntos de encuentro entre la academia y el saber popular

Hernando Carvajalino-Bayona

Pág. 112 ES

Arquitectura, modernidad, modernización

$$
\begin{aligned}
& \text { Jean-Louis Cohen } \\
& \text { Traductores } \\
& \text { Andrés Avila-Gómez. } 126 \\
& \text { Diana Carolina Ruiz } \\
& \text { ES }
\end{aligned}
$$


Arquitecturas colectivas y participación como estrategias para la

Arquiteturas coletivas e participação como estratégias para construir a cidad latino-americana

\section{Stella Díaz-Osorio}

La caminabilidad en Bogotá: propósitos y condiciones socioespaciales que facilitan y limitan esta experiencia

The Walkability of Bogotá: purposes and socio-spatial conditions that facilitate and limit this experience

A caminhabilidade em Bogotá: propósitos e condições socioespaciais que facilitam e limitam essa experiência

\section{Andrea Burbano}

Planificación comunitaria en barrios socialmente vulnerables.

Identificación de los actores sociales en una comunidad

Community planning in socially vulnerable neighborhoods.

Identification of social actors in a community

Planejamento comunitário em bairros socialmente vulneráveis.

Identificação dos atores sociais em uma comunidade

Rafael Alejandro Tavares-Martínez

esús Manuel Fitch-Osuna

Desvanecimiento de la frontera como límite. Imaginario del borde como espacio público físico y virtual

$m$

迹

Durabilidad de los materiales naturales de construcción: percepciones de proyectistas, constructores y usuarios en Florianópolis, Brasil

Durability of natural building materials: Perceptions of designers, builders, and users in Florianópolis, Brazil

Durabilidade dos materiais naturais de construção: percepções de projetistas, construtores e usuários em Florianópolis, Brasil

\section{Zuleica Maria Patricio-Karnopp}

Lisiane Ilha-Librelotto

Thermal comfort in buildings for wet processing of coffee

\section{Confort térmico en edificaciones para procesamiento húmedo de café}

Conforto térmico em instalaçōes para processamento úmido de café

\section{Ida de Fátima Ferve-Tartióco}

airo Alexander Osorio-Saraz

Robinson Osorio-Hernandez

La arquitectura en los barrios: puntos de encuentro entre la academia y el saber popular

Architecture in neighborhoods: meeting points between academia and popular knowledge

A arquitetura nos bairros: pontos de encontro entre a academia e o saber popular Hernando Carvajalino-Bayona

\section{Arquitectura, modernidad, modernización}

Architecture, modernity, modernization

Arquitetura, modernidade, modernização
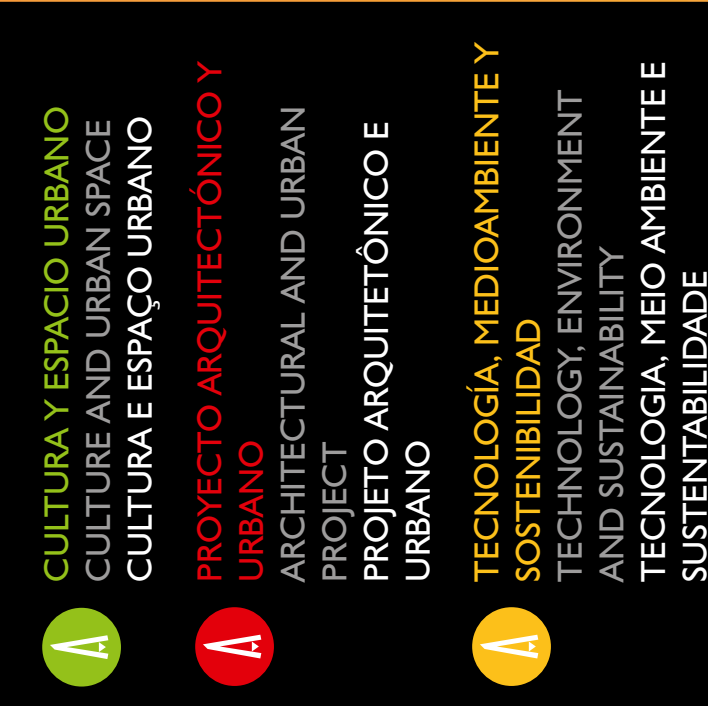

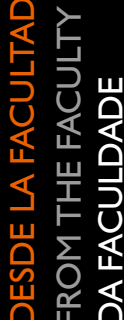

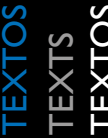
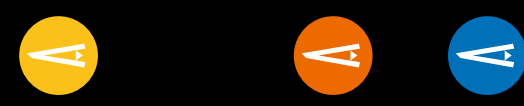

1) Clarivate

publind

Clarivate
Analytics

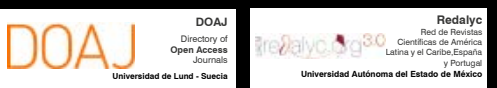

n.

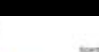

REDIB

EBSCO

cis

latindex unmoxom

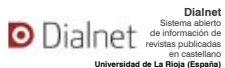

LatinREV

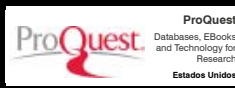

MIคR

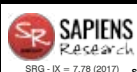

Goosin

Google

ARLA

hi. https://www.mendeley.com/profles/revista-de-arquitectura-bogot/

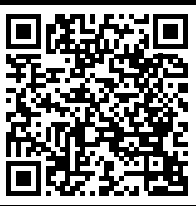

ISSN: 1657-0308 